\title{
Experimental reconstruction of photon statistics without photon counting
}

\author{
Guido Zambra, Alessandra Andreoni, Maria Bondan** \\ Istituto Nazionale per la Fisica della Materia, INFM and Dipartimento di Fisica e Matematica, \\ Università degli Studi dell'Insubria, Como, Italia. \\ Marco Gramegna, Marco Genoves $₫$ and Giorgio Brida \\ Istituto Elettrotecnico Nazionale, IEN, Galileo Ferraris, Torino, Italia. \\ Andrea Rossi and Matteo G. A. Paris \\ Dipartimento di Fisica dell' Università di Milano, Italia.
}

\begin{abstract}
Experimental reconstructions of photon number distributions of both continuous-wave and pulsed light beams are reported. Our scheme is based on on/off avalanche photodetection assisted by maximum-likelihood estimation and does not involve photon counting. Reconstructions of the distribution for both semiclassical and quantum states of light are reported for single-mode as well as for multimode beams.

PACS numbers: 42.50.Ar, 42.50.Dv, 03.65.Wj
\end{abstract}

The measurement of the statistical distribution of the number of photons provides fundamental information on the nature of any optical field. The choice of a detector with internal gain suitable for the measurement is not trivial when the flux of the photons to be counted is such that more than one photon is detected in the timewindow of the measurement, which is set by the detector pulse-response, or by an electronic gate on the detector output, or by the duration of the light pulse. In this case, we need a congruous linearity in the internal current amplification process: each of the single electrons produced by the different photons in the primary step of the detection process (either ionization or promotion to a conduction band) must experience the same average gain and this gain must have sufficiently low spread. The fulfillment of both requisites is necessary for the charge integral of the output current pulse be proportional to the number of detected photons. Photon detectors that can operate as photon counters are rather rare. Among these, PhotoMultiplier Tubes (PMT's) [1] and hybrid photodetectors 2] have the drawback of a low quantum efficiency, since the detection starts with the emission of an electron from the photocathode. Solid state detectors with internal gain, in which the nature of the primary detection process ensures higher efficiency, are still under development. Highly efficient thermal detectors have also been used as photon counters, though their operating conditions are still extreme (cryogenic conditions) to allow common use [3, 4]. The advent of quantum tomography provided an alternative method to measure photon number distributions 5]. However, the tomography of a state, which has been applied to several quantum states $[\underline{6}$, needs the implementation of homodyne detection, which in turn re-

\footnotetext{
*Electronic address: maria.bondani@uninsubria.it

$\dagger$ Electronic address: genovese@ien.it

‡Electronic address: matteo.paris@fisica.unimi.it
}

quires the appropriate mode matching of the signal with a suitable local oscillator at a beam splitter. Such mode matching is a particularly challenging task in the case of pulsed optical fields.

Photodetectors that are usually employed in quantum optics such as Avalanche PhotoDiodes (APD's) operating in the Geiger mode [4, 7] seem to be by definition useless as photon counters. They are the solid state photodetectors with the highest quantum efficiency but they have the obvious drawback that the breakdown current is independent of the number of detected photons, which in turn cannot be determined. The outcome of these APD's is either "off" (no photons detected) or "on" i.e. a "click", indicating the detection of one or more photons. Actually, such an outcome can be provided by any photodetector (PMT, hybrid photodetector, cryogenic thermal detector) for which the charge contained in dark pulses is definitely below that of the output current pulses corresponding to the detection of at least one photon. Note that for most high-gain PMT's the anodic pulses corresponding to no photons detected can be easily discriminated by a threshold from those corresponding to the detection of one or more photons.

The statistics of the "no-click" and "click" events from an on/off detector, assuming no dark counts, is given by

$$
p_{0}(\eta)=\sum_{n}(1-\eta)^{n} \varrho_{n},
$$

and $p_{>0}(\eta)=1-p_{0}(\eta)$, where $\varrho_{n}$ is the probability of finding $n$ photons and $\eta$ is the quantum efficiency of the detector, i.e. the probability of a single photon to be revealed. At first sight the statistics of an on/off detector appears to provide quite a scarce piece of information about the state under investigation. However, if the statistics about $p_{0}(\eta)$ is collected for a suitably large set of efficiency values then the information is enough to reconstruct the whole photon distribution $\varrho_{n}$ of the signal, upon a suitable truncation of the Hilbert space. 
The reconstruction of photon distribution through on/off detection at different efficiencies has been analyzed [8] and its statistical reliability investigated in some details [9]. In addition, the case of few and small values of $\eta$ 10] has been addressed. However, whilst these theoretical studies found an application to realize a multichannel fiber loop detector 11, 12, an experimental implementation of this technique for reconstructing photon distribution of a free-propagating field is still missing. In view of the relevance of photon distribution for applications in quantum information and foundations of quantum mechanics, the purpose of this letter is to show that a reconstruction of the photon distribution by using this technique can be effectively realized experimentally with excellent results both for free-propagating continuous-wave (cw) and pulsed light beams, for both single-mode semiclassical and quantum states, as well as for multimode states.

The procedure consists in measuring a given signal by on/off detection using different values $\eta_{\nu}(\nu=1, \ldots, K)$ of the quantum efficiency. The information provided by experimental data is contained in the collection of frequencies $f_{\nu}=f_{0}\left(\eta_{\nu}\right)=n_{0 \nu} / n_{\nu}$ where $n_{0 \nu}$ is the number of "no click" events and $n_{\nu}$ the total number of runs with quantum efficiency $\eta_{\nu}$. Then we consider expression (11) as a statistical model for the parameters $\varrho_{n}$ to be solved by maximum-likelihood (ML) estimation. Upon defining $p_{\nu} \equiv p_{0}\left(\eta_{\nu}\right)$ and $A_{\nu n}=\left(1-\eta_{\nu}\right)^{n}$ we rewrite expression (11) as $p_{\nu}=\sum_{n} A_{\nu n} \varrho_{n}$. Since the model is linear and the parameters to be estimates are positive (LINPOS problem), then the solution can be obtained by using the Expectation-Maximization algorithm (EM) 13]. By imposing the restriction $\sum_{n} \varrho_{n}=1$, we obtain the iterative solution

$$
\varrho_{n}^{(i+1)}=\varrho_{n}^{(i)} \sum_{\nu=1}^{K} \frac{A_{\nu n}}{\sum_{\lambda} A_{\lambda n}} \frac{f_{\nu}}{p_{\nu}\left[\left\{\varrho_{n}^{(i)}\right\}\right]}
$$

where $p_{\nu}\left[\left\{\varrho_{n}^{(i)}\right\}\right]$ are the probabilities $p_{\nu}$, as calculated by using the reconstructed distribution $\left\{\varrho_{n}^{(i)}\right\}$ at the $i$-th iteration. As a measure of convergence we use the total absolute error at the $i$-th iteration $\varepsilon^{(i)}=$ $\sum_{\nu=0}^{K}\left|f_{\nu}-p_{\nu}\left[\left\{\varrho_{n}^{(i)}\right\}\right]\right|$ and stop the algorithm as soon as $\varepsilon^{(i)}$ goes below a given level. The total error measures the distance of the probabilities $p_{\nu}\left[\left\{\varrho_{n}^{(i)}\right\}\right]$, as calculated at the $i$-th iteration, from the actual experimental frequencies. As a measure of accuracy we adopt the fidelity $G^{(i)}=\sum_{n} \sqrt{\varrho_{n} \varrho_{n}^{(i)}}$ between the reconstructed distribution and the theoretical one.

In order to verify the potentialities of this technique we applied it to the reconstruction of various quantum optical states, generated either in the cw or in the pulsed regimes. As a first example we have considered single photon states that have been generated by producing Parametric Down Conversion (PDC) heralded photons. In little more detail, a pair of correlated photons has been generated by pumping a type II $\beta$-Barium-Borate

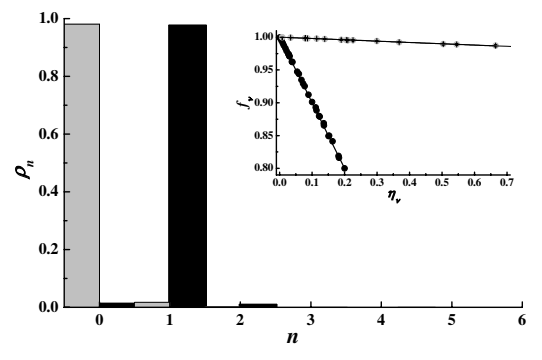

FIG. 1: Reconstruction of the photon distribution for a weak coherent state (grey) and for the heralded single-photon state produced in type II PDC (black). Inset: Experimental frequencies $f_{\nu}$ of no-click events as a function of the quantum efficiency $\eta_{\nu}$ for a weak coherent state (upper curve) and for PDC heralded photon state (lower curve) compared with the theoretical curves, $p_{\nu} \simeq 1-\eta_{\nu}|\alpha|^{2}$ and $p_{\nu}=1-\eta_{\nu}$ respectively.

(BBO) crystal with a cw Argon ion laser beam (351 nm) in collinear geometry. After having split the photons of the pair by means of a polarizing beam splitter, the detection of one of the two by a silicon avalanche photodiode detector (SPCM-AQR-15, Perkin Elmer) was used as an indication of the presence of the second photon in the other channel, namely a window of 4.9 ns was opened for detection in arm 2 in correspondence to the detection of a photon in arm 1. This "heralded photon" was then measured by a silicon avalanche photodiode detector (SPCM-AQR-15, Perkin Elmer) preceded by an iris and an interference filter (IF) at $702 \mathrm{~nm}, 4 \mathrm{~nm}$ FWHM, inserted with the purpose of reducing the stray light. The quantum efficiency of the detection apparatus (including IF and iris) was measured to be $20 \%$ by using the PDC calibration scheme (see 14]). Lower quantum efficiencies were simulated by inserting calibrated neutral optical filters on the optical path. A comparison of the observed frequencies $f_{\nu}$ with the theoretical curve $\left(1-\eta_{\nu}\right)$ is presented in the inset of Fig. [1 The photon distribution has been reconstructed using $K=34$ different values of the quantum efficiency from $\eta_{\nu} \simeq 0$ to $\eta_{\nu} \simeq 20 \%$ with $n_{\nu}=10^{6}$ runs for each $\eta_{\nu}$. Results at iteration $i=10^{6}$ are shown in Fig. 11 As expected, the PDC heralded photon state largely agrees with a single photon Fock state. However, also a small two photons component and a vacuum one are observed. The $\rho_{2}$ contribution is expected, by estimating the probability that a second photon randomly enters the detection window, to be $1.85 \%$ of $\rho_{1}$, in agreement with what observed. A non zero $\rho_{0}$ is also expected due to background. This quantity can be evaluated to correspond to $(2.7 \pm 0.2) \%$ by measuring the counts when the polarization of the pump beam is rotated to avoid generation of parametric fluorescence. Also this estimate is in good agreement with the reconstructed $\rho_{0}$. A second example is represented by a strongly attenuated coherent state, which has been produced by a He-Ne laser beam attenuated to photoncounting regime by insertion of neutral filters. Also in 
this case the same silicon avalanche photodiode detector was used. The reconstructed distribution (with $K=15$ different values of the quantum efficiency from $\eta_{\nu} \simeq 0$ to $\eta_{\nu} \simeq 66 \%$ with $n_{\nu}=10^{6}$ runs for each $\eta_{\nu}$ ) agrees well with what expected for a coherent state with average number of photons $|\alpha|^{2} \simeq 0.02$. In the inset of Fig. 1 the frequencies $f_{\nu}$ as a function of $\eta_{\nu}$ are compared with the theoretical prediction $p_{\nu}=\exp \left\{-\eta_{\nu}|\alpha|^{2}\right\} \simeq 1-\eta_{\nu}|\alpha|^{2}$. Notice that in this case we do not have IF or irises in front of the detector and all the other attenuations can be included in the generation of the state: thus the highest quantum efficiency is taken to be $66 \%$ as declared by the manufacturer data-sheet for the photodetector.

In the pulsed domain, we have measured three different optical states generated starting from the third harmonics (349 nm, $4.45 \mathrm{ps)} \mathrm{of} \mathrm{a} \mathrm{cw} \mathrm{mode-locked} \mathrm{Nd:YLF} \mathrm{laser}$ regeneratively amplified at a repetition rate of $500 \mathrm{~Hz}$ (High Q Laser). For all the measurements, the light was delivered to a photo multiplier tube (PMT, Burle 8850) through a multi mode fiber (100 $\mu \mathrm{m}$ core diameter). Although the PMT has the capability of counting the number of photoelectrons produced by one or more photons 1], for the present application we used it in a Geigerlike configuration, by setting a threshold to discriminate on/off events. Furthermore, by using the detector in the regime of linear response, the knowledge of its photocathode quantum efficiency is sufficient ti determine the $\eta_{\nu}$ values.

The first measurement was performed on the pulse emerging from the laser source. Due to the pulsed nature of the source, we do not expect to recover a true Poissonian statistics. Rather, we expect a Gaussian distribution $\varrho_{n, G}$ [17] with mean value $N$ and variance $N+\sigma^{2}$ which takes into account the presence of noise. $N$ is the photon mean value and $\sigma^{2} / N$ is as a measure of the deviation from Poissonian statistics. In Fig. 2]a) we show the photon distribution $\varrho_{n}$, reconstructed at the $i=50000$ iteration of the ML algorithm, along with the best fit obtained with the model $\varrho_{n, G}$ (fitting parameters $N=4.88$ and $\left.\sigma^{2}=0.63\right)$. The inset of the figure compares the experimental frequency $f_{\nu}$ data $(K=37$ values of $\eta$, $n_{\nu}=10^{4}$ runs for each $\eta$ ) as a function of $\eta_{\nu}$ with the theoretical values calculated through (11) and the parameters given by the fit of the photon distribution. Both the reconstructed distribution and the experimental frequencies agrees very well with the above Gaussian model. The fidelity of the reconstruction is $G \simeq 0.998$. Using the estimated value of $\sigma^{2}$, a deviation of about $13 \%$ from the Poissonian statistics can be derived for the laser photon number distribution.

A second measurement was performed on laser pulses diffused by a moving ground glass. If the photons are collected from within an area of spatial coherence, the system acts as a pseudo-thermal source, whose photon number distribution is given by $\varrho_{n, T}=N^{n}(N+1)^{-n-1}$. Figure 2 b) shows the photon distribution $\varrho_{n}$, as reconstructed at the $400 t h$ iteration and the best fit of the data with $\varrho_{n, T}(N=5.33)$; the fidelity is given by $G \simeq 0.995$.
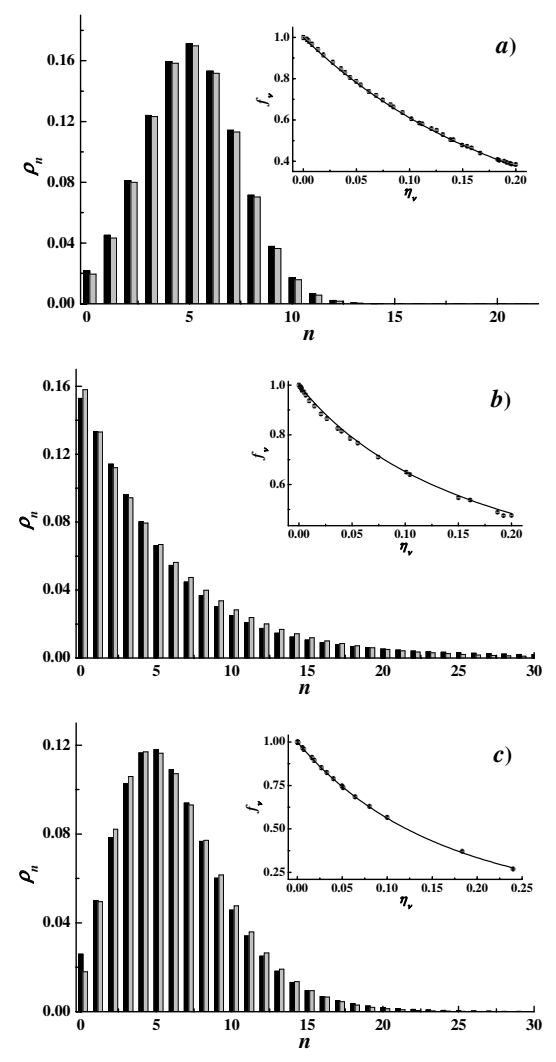

FIG. 2: Reconstructed photon distribution (black bars) and best theoretical fit (grey bars) for three different states in the pulsed regime: a) laser pulse, b) diffused laser pulse, c) multimode state produced in type I PDC. Insets: Experimental frequency $f_{\nu}$ data in function of the quantum efficiency $\eta_{\nu}$ and theoretical model for each one of the states.

The inset of the figure contains the experimental frequency $f_{\nu}$ data $\left(K=24\right.$ values of $\eta, n_{\nu}=10^{4}$ runs for each $\eta$ ) and their theoretical values as calculated from (11).

The last measurement was performed on the blue portion $(420 \mathrm{~nm})$ of the down conversion fluorescence produced by a type I BBO crystal $(10 \mathrm{~mm}$ depth, cut at $34 \mathrm{deg}$ ) pumped by the laser pulse. The pump, incident orthogonally to the crystal face, had an intensity $\sim 60$ $\mathrm{GW} / \mathrm{cm}^{2}$. In this experimental condition we expect a coherence time of the generated field of $\sim 1 \mathrm{ps}$, that corresponds to measuring a convolution of 4-5 temporal modes [15]. The photon number distribution is expected to be a multithermal distribution of the form

$$
\varrho_{n, M}=\frac{(n+\mu-1) !}{n !(\mu-1) !(1+N / \mu)^{n}(1+\mu / N)^{\mu}},
$$

where $\mu$ is the number of temporal modes. The photon distribution reconstructed at the $i=1500$ iteration, is shown in Fig. 2 c) along with the best fit of the data using (3) $(N=6.17$ and $\mu=5)$; the fidelity of the reconstruction is given by $G \simeq 1$. In the inset of the figure we show the experimental frequency $f_{\nu}$ data $(K=18$ val- 
ues of $\eta, n_{\nu}=10^{4}$ runs for each $\eta$ ) and their theoretical values as calculated according to (1).

As a comment to the experimental results in the pulsed regime, we note that the best reconstruction of the photon distribution is achieved at a different number of iterations for the three different measured optical states, and that the absolute error $\varepsilon$ does not approach the same value. This is due to the presence of excess noise in our measurements, since the stability and the repetition rate of our source $(500 \mathrm{~Hz})$ limits to $n_{\nu} \sim 10^{4}$ the number of runs for each value of the quantum efficiency [9]. The choice of the best iteration to stop the algorithm is driven by the possibility to fit the distribution with a suitable model. We stress that there was no a-priori decision in choosing a Gaussian distribution for case a) or of a multithermal distribution for case c), but, on the contrary, we followed the a-posteriori observation that no other distribution could fit equally well the reconstructed data.

As to the comparison of the present technique with other schemes to reconstruct the photon distribution we have to distinguish between $\mathrm{cw}$ and pulsed regime. For cw field an alternative technique is represented by quantum homodyne tomography (QHT), which has indeed been applied to the reconstruction of single-photon and single-photon added states of light [18]. The advantage of our technique compared to QHT is twofold. On one hand QHT requires, for the same task, a more complex apparatus and high-efficiency photodetectors. On the other hand, QHT is more noisy. In fact, homodyne data contains the whole information about the state under inves- tigation (not only the photon distribution) and, in turn, this results in a more noisy determination when only part of the information is of interest [19]. In order to obtain results such those of Fig. 11 QHT requires a by far larger set of data. In the pulsed regime, where realization of QHT is still challenging, the direct measurement of the photon statistics can be conveniently done by PMT's or hybrid photodetectors [1, 2]. However, owing to limitations of the values of the maximum detection efficiency, results at number of photons as low as those in Fig. 2 cannot be obtained by PMT's and are at limit of feasibility for hybrid photodetectors. While our method is particularly suited to measure the photon distributions of low-intensity field, where the above detectors are not effective, it can be always applied to higher intensity fields.

In conclusion, we experimentally implemented a reconstruction method for the photon distribution based on on/off detection at different quantum efficiency followed by a ML iterative algorithm. Our experimental results demonstrate that the technique can be applied to both $\mathrm{cw}$ and pulsed regimes, and for a wide range of signal energy (from single-photon states to mesoscopic signals), a feature that makes it preferable to other methods till now devised for reconstructing photon number distribution.

This work has been supported by MIUR (FIRB RBAU01L5AZ-002 and RBAU014CLC-002), by INFM (PRA-CLON) and by "Regione Piemonte". MGAP thanks Zdenek Hradil, Alessandro Ferraro and Stefano Olivares for useful discussions.
[1] G. Zambra, M. Bondani, A. S. Spinelli, A. Andreoni, Rev. Sci. Instrum. 75, 2762 (2004).

[2] E. Hergert, Single Photon Detector Workshop, Gaithersburg, NIST (2003).

[3] J. Kim, S. Takeuchi, Y. Yamamoto, and H.H. Hogue, Appl. Phys. Lett. 74, 902 (1999); A. Peacock, P. Verhoeve, N. Rando, A. van Dordrecht, B. G. Taylor, C. Erd, M. A. C. Perryman, R. Venn, J. Howlett, D. J. Goldie, J. Lumley, and M. Wallis, Nature 381, 135 (1996).

[4] G. Di Giuseppe, A. V. Sergienko, B. E. A. Saleh, and M. C. Teich in Quantum Information and Computation, E. Donkor, A. R. Pirich, and H. E. Brandt Eds., Proceedings of the SPIE 5105, 39 (2003).

[5] M. Munroe, D. Boggavarapu, M. E. Anderson, and M. G. Raymer, Phys. Rev. A 52, R924 (1995); Y. Zhang, K. Kasai, and M. Watanabe, Opt. Lett. 27, 1244 (2002).

[6] M. Raymer, M. Beck in Quantum states estimation, M. G .A Paris and J. Řeháček Eds., Lect. Not. Phys. 649 (Springer, Berlin-Heidelberg, 2004).

[7] F. Zappa, A. L. Lacaita, S. D. Cova, and P. Lovati, Opt. Eng. 35, 938 (1996); D. Achilles, C. Silberhorn, C. Śliwa, K. Banaszek, and I. A. Walmsley, Opt. Lett. 28, 2387 (2003).

[8] D. Mogilevtsev, Opt. Comm 156, 307 (1998); Acta Phys. Slov. 49, 743 (1999).
[9] A. R. Rossi, S. Olivares, and M. G. A. Paris, Phys. Rev. A 70, 055801 (2004).

[10] A. R. Rossi and M. G. A. Paris, Eur. Phys. J. D 32, 223 (2005).

[11] J. Řeháček, Z. Hradil, O. Haderka, J. Peřina, Jr., and M. Hamar, Phys. Rev. A 67, 061801(R) (2003); O. Haderka, M. Hamar, J. Peřina, Eur. Phys. Journ. D 28, (2004).

[12] K. Banaszek, I. A. Walmsley, Opt. Lett. 28, 52 (2003).

[13] A.P. Dempster, N.M. Laird, D.B. Rubin, J. R. Statist. Soc. B 39, 1 (1977); Y. Vardi and D. Lee, J. R. Statist. Soc. B 55, 569 (1993); R. A. Boyles, J. R. Statist. Soc. B 45, 47 (1983).

[14] G. Brida, M. Genovese, and C. Novero, J. Mod. Opt. 47, 2099 (2000), and Refs. therein.

[15] F. Paleari, A. Andreoni, G. Zambra, M. Bondani. Opt. Expr. 13, 2816 (2004).

[16] G. F. Knoll, Radiation detection and measurement. Second Edition (John Wiley \& Sons, New York, 1989).

[17] R. Loudon, The Quantum Theory of Light, (Oxford University Press, New York, 2000).

[18] A. Zavatta, S. Viciani, M. Bellini, Science 306, 660 (2004).

[19] G. M. D'Ariano, M. G. A. Paris, Phys. Lett. A 23349 (1997). 